\title{
Identification of batik making method from images using convolutional neural network with limited amount of data
}

\author{
Mohammad Arif Rasyidi, Ruktin Handayani, Fauzul Aziz \\ Department of Informatics, Universitas Internasional Semen Indonesia, Indonesia
}

\begin{tabular}{l} 
Article Info \\
\hline Article history: \\
Received Dec 4, 2020 \\
Revised Mar 29, 2021 \\
Accepted Apr 6, 2021 \\
\hline
\end{tabular}

\section{Keywords:}

Batik

Batik cap

Batik tulis

Classification

Convolutional neural network

Transfer learning

\begin{abstract}
This study aims to apply the convolutional neural network (CNN) to classify batik based on its manufacturing method, namely Batik Tulis which are hand drawn, Batik Cap where stamps are used to create the pattern, and Batik Printing which are printed using textile printing machine. We collected 40 images for each type of batik with a total of 120 images. To speed up and simplify the model building process, we implemented transfer learning with 3 basic CNN model architectures, namely ResNet, DenseNet, and VGG with batch normalization. We also experimented with building a new dataset by breaking each image down into 30 smaller images. Image augmentation was also used to prevent overfitting as well as to provide variations in the training data. The experimental results with 5-fold cross validation show that densenet169 gives the best results on the original dataset with an accuracy of $79.17 \%$ while vgg 13 bn shows the best performance on the modified dataset with an accuracy of $87.61 \%$. All models showed an increase in performance when using the modified dataset, except densenet 169 which did not show a significant difference in performance.
\end{abstract}

This is an open access article under the CC BY-SA license.

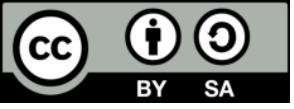

\section{Corresponding Author:}

Mohammad Arif Rasyidi

Department of Informatics

Universitas Internasional Semen Indonesia

Jl. Veteran, Gresik, Jawa Timur 61122, Indonesia

Email: mohammad.rasyidi@uisi.ac.id

\section{INTRODUCTION}

Since it was first created, perhaps hundreds to thousands of years ago, until it was named Masterpieces of the Oral and Intangible Heritage of Humanity by UNESCO in 2009 [1] and to this day, batik, a patterned cloth from Indonesia, has undergone many developments. Traditionally the process of making batik begins with drawing the desired design pattern on the fabric using a pencil. The manufacturing process is continued by applying liquid wax on both sides of the cloth according to the previously formed pattern using canting as shown in Figure 1. Canting is a pen-like instrument which consists of bamboo handle and copper wax pot with a narrow pipe spout. Next, the fabric is dyed by dipping it in a dye solution. In this process, the part of the cloth that has been covered with wax before will not get wet, so that the original color is maintained. The wax is then removed by scraping it with a knife or other metal object. The cloth is then washed and boiled to remove any lingering wax residue. The process of waxing, coloring, and removing wax was repeated for each required color combination [2], [3]. Because the batik maker draws the design pattern using the canting by hand, the batik produced by this method is called Batik Tulis, which comes from the Javanese language for "writing". Due to the complexity of the process, the process of making Batik Tulis takes a long time. It can take 30 to 50 days or even months for producing a piece of cloth [2], [3]. 
Around 1840, a method of making batik using cap (stamp) was developed to replace canting [3]. A cap is a block-shaped printing tool made of metal such as copper with a batik pattern. With a cap, the batik maker can apply the entire batik pattern with one application as shown in Figure 1. This greatly accelerates the process of making batik from the previous method. Additionally, in general, the resulting design patterns are tidier than manual work using canting. However, unlike Batik Tulis which has a color pattern on both sides, most Batik Cap only has a color on one side of the fabric.

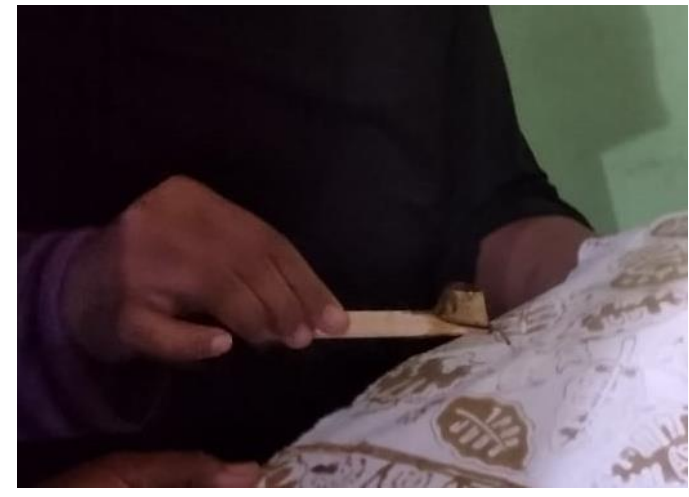

(a)

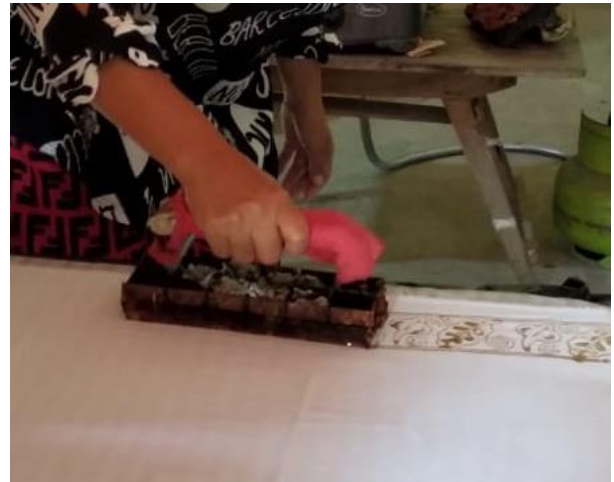

(b)

Figure 1. The process of making batik using tulis, (a) And cap, (b) Method

Along with technological developments and the need for more affordable batik, a new modern variation emerged, namely Batik Printing. Unlike the two previous variants, Batik Tulis and Batik Cap, which are produced using wax, the Batik Printing production process does not require this. The entire manufacturing process of Batik Printing is carried out using a textile machine, which often raises debate as to whether the fabric with the resulting batik pattern can be called batik.

There is no doubt that the variation in the method of producing batik creates a difference in the price of the batik fabric made. Batik produced by the hand drawn method (tulis) usually has a much higher market value than batik fabric produced by other methods due to the length of the production process and the artistic value it displays. While it can be easily identified visually by a skilled person, often it is difficult for average people to find out how the batik is produced because of their similar design patterns. Furthermore, in the online trade sector, buyers can only see photos of the batik cloths being sold without being able to hold and inspect them directly, which is prone to misidentification and can lead to fraud.

To solve this problem, in this study we propose a supervised learning approach by building a classification model to help ordinary people identify methods of making batik. The classification model for the batik making method will be built using the convolutional neural network (CNN) and transfer learning. $\mathrm{CNN}$ is a special type of deep learning which is very often applied to the image processing field. Unlike traditional machine learning methods which require a feature extraction process from images prior to model training, CNN incorporates the process into the training phase, and thus does not require a separate feature extraction step. This approach is what makes it so powerful and popular today. Some examples of CNN applications include fashion image classification [4], classification of retinal tomographic images [5], and handwriting recognition [6].

One of the challenges we faced in building such a classification model was the insufficient amount of labeled data that we were able to collect. However, as we all know, deep learning methods such as CNN require a sufficient amount and variety of data to be able to produce high-quality models. To overcome this problem, the approach we take is to take advantage of CNN models that have been previously developed by researchers for other problems and adapt them for classifying batik making method. This technique is often known as transfer learning. With several proven and readily available CNN models, such as AlexNet [7], MobileNet [8], [9], ResNet [10], DenseNet [11], SqueezeNet [12], and VGG [13], it is possible to create new CNN models based on these models so that the training phase can be carried out more rapidly and easily.

In the literature, there have been many previous studies related to batik, for example, [14]-[28]. Most of these studies concentrate more on the feature extraction aspect of the classification. Generally, in the image recognition process, the features generated from the feature extraction process are used as input to train prediction models using traditional machine learning algorithms. Although this approach is very 
popular, with the advent of deep learning, especially with $\mathrm{CNN}$, new classification methods that do not require manual feature extraction are starting to be widely applied to batik classification problems [29]-[34]. However, despite the vast number of studies related to batik, only a few studies have focused on predicting batik-making methods, with others more frequently aimed for batik pattern classification. One of the studies we found that focuses on the prediction of the batik making method is [24] which utilizes the gray level cooccurrence matrix (GLCM) for the feature extraction process from the image and learning vector quantization (LVQ) for classification. Even though the resulting performance of this study is quite high with almost 99\% accuracy, the study uses even less data than what we used in this study, namely 10 images for each type of batik. Each image is then split into 135 images and then randomly divided into training and test size with a ratio of 2:1. The weakness of the method used is that because each image is broken down into smaller images before being divided into training and test data, the fractions of the same image will be scattered partly in the training data and partly in the test data. Thus, when there are some parts in the training data that are exactly the same or very similar to some images in the test set, the result will be misleading as the model may not have learned anything useful to classify new images. As the images come from the same source image, the probability of this happening is very high, so its applicability and result may need to be reviewed.

Accordingly, our contributions are as follows. We are one of the few who focus on predicting batik making methods and not on batik patterns. To the best of our knowledge, we are also the first apply CNN to classify batik making methods, albeit using only small amount of data. Finally, we also experimented by splitting our images in building the model, which is similar to the approach of [24]. However, we made improvements in the experimental design where we only split the image after dividing it into training and test sets. Thus, it is expected that the resulting model performance measurement results can be more accurate.

\section{RESEARCH METHOD}

As shown in Figure 2, our research methodology mainly consists of five main processes: data collection, cross validation, image augmentation, model training, and testing.

\subsection{Data collection}

We collected a total of 120 images of batik, which consist of batik tulis, cap, and printing (40 images for each type of batik). Each of the collected images has been cropped and resized to 540x630 pixels. Some examples of batik images are shown in Figure 3.

\subsection{Cross validation}

Due to the small amount of data collected, the test method using an ordinary holdout set may cause high bias and variation in the performance being measured. Therefore, in this study, we used the 5 -fold cross validation method to overcome this. In 5 -fold cross validation, the dataset is divided randomly into 5 parts called folds with each fold containing 24 images ( 8 images for each type of batik). Then, alternately each fold is used as a test-set while the other 4 folds will be used as training data to build a model that will be tested on the test fold as illustrated in Figure 1. The five test results obtained in each iteration will then be averaged to get the final performance.

Apart from using the original data directly, we also experimented by splitting each image into 30 different images with a size of $180 \times 180$ pixels. In this image splitting process, we first divide the image into 42 blocks, each measuring 90x90 pixels. Next, we formed new images by taking $2 \times 2$ blocks starting from the top left corner then shifting them to the right and down by 1 block. This is similar to the convolution and pooling operations on CNN with a filter size of $2 \times 2$ and stride 1 . This process is illustrated in Figure 4. From this process, we obtained 720 images for each fold for a total of 3600 images. The reason we do the image splitting process after dividing the data into 5 folds and not before is so that the results of dividing each image are not separated in training and test sets which might cause the measured performance to be less reliable. When using the model built using this dataset in production, because the original image is divided into 30 smaller images, we will of course get 30 different predictions. The final prediction result for the original image can thus be obtained simply by a majority vote.

\subsection{Model training}

With the small amount of data we have collected, the trained model will be prone to overfitting. Like our previous research [34], image augmentation is used to overcome this problem. In the training process, in each iteration, the images given to the model will be altered with a small random transformation without losing their original content. The transformations used in this study include horizontal and vertical flip, rotation, zoom, lighting, and symmetric wrap. Table 1 shows the parameters of each transformation. 

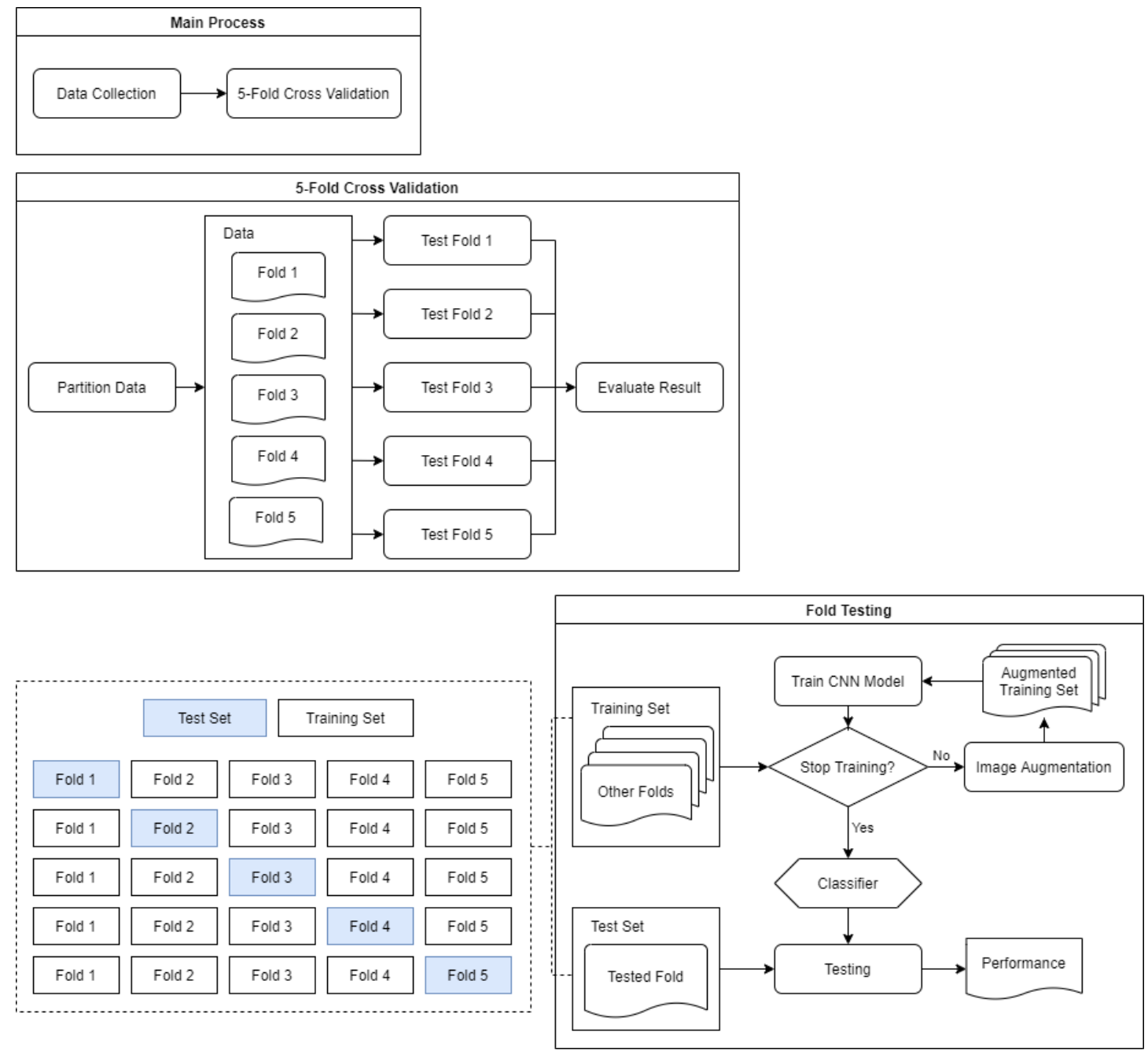

Figure 2. Research method

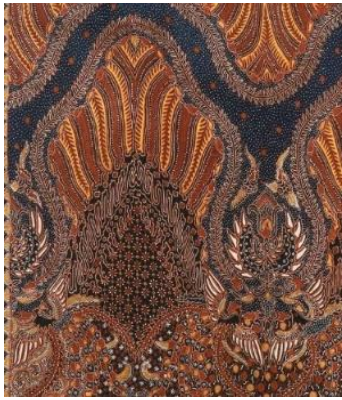

(a)

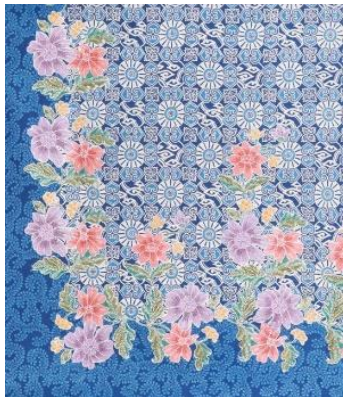

(b)

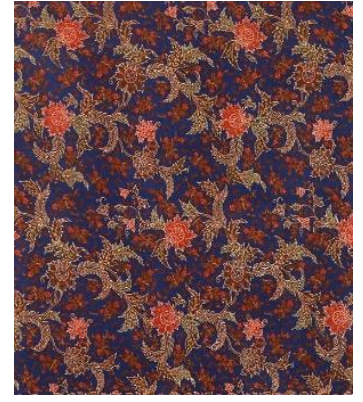

(c)

Figure 3. Some examples of images used in this study, (a) Batik Tulis, (b) Batik Cap, (c) Batik Printing

\subsection{Image augmentation}

As previously stated, the model building was carried out using $4 / 5$ parts of the total data. From this data, we further divide it into 3: 1 with 3 parts used as training data while the remaining 1 part is used for data validation. Validation data is used to determine when the training process must be stopped so that the resulting model does not overfit the training data.

There are 3 types of $\mathrm{CNN}$ architectures and their variations that we use, namely ResNet (resnet18, resnet34, resnet50, resnet101 and resnet152) [10], DenseNet (densenet121, densenet169, densenet201 and densenet161) [11], and VGG with batch normalization (vgg11_bn, vgg13_bn, vgg16_bn and vgg19_bn) [13]. The training process is carried out using these basic models which have been previously trained on the

\footnotetext{
Identification of batik making method from images using convolutional neural... (Mohammad Arif Rasyidi)
} 
ImageNet dataset and modified to accommodate the identification problem of the batik making method. To speed up the training process, each model is trained using the 1cycle policy [35]. In addition, early stopping is also applied to avoid overfitting.
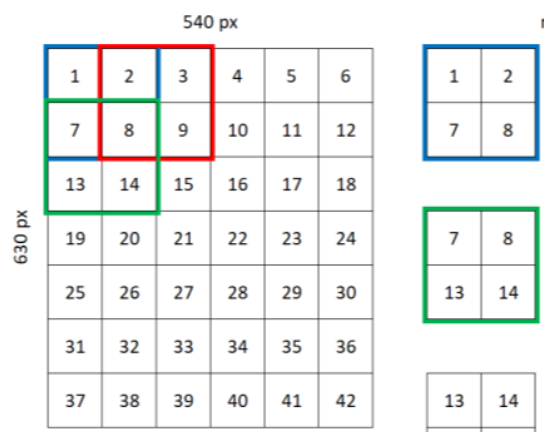

result $=30$ images $(180 \times 180 \mathrm{px})$
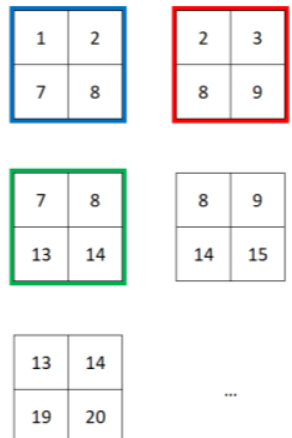

Figure 4. Illustration of image splitting process
Table 1. Parameters for image

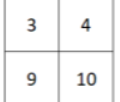
augmentation

\begin{tabular}{cc}
\hline Transformation & Value \\
\hline Horizontal Flip & True/False \\
Vertical Flip & True/False \\
Max Rotation & $45^{\circ}$ \\
Max Zoom & 1.3 \\
Max Lighting & 0.3 \\
Max Symmetric Warp & 0.2
\end{tabular}

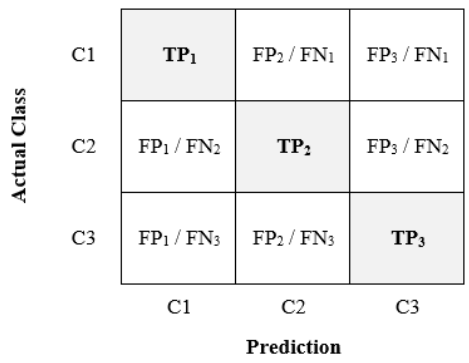

Figure 5. Sample confusion matrix, here $\mathrm{C} 1$, C2, and C3 represent Batik Tulis, Batik Cap, and Batik Printing respectively

\subsection{Testing}

Testing is done by applying trained models to the test data. Confusion matrix as shown in Figure 5 is then used to summarize the result of the test. There are four criteria tested, namely accuracy, precision, recall, and F-measure which will be calculated according to the following formulas;

$$
\begin{aligned}
& \text { accuracy }=\frac{\sum_{i=1}^{k} T P_{i}}{n} \\
& \text { precision }=\frac{\sum_{i=1}^{k} \frac{T P_{i}}{T P_{i}+F P_{i}}}{k} \\
& \text { recall }=\frac{\sum_{i=1}^{k} \frac{T P_{i}}{T P_{i}+F N_{i}}}{k} \\
& \text { F-measure }=\frac{\sum_{i=1}^{k} \frac{2 T P_{i}}{2 T P_{i}+F P_{i}+F N_{i}}}{k}
\end{aligned}
$$

where; $\mathrm{TP}=$ true positive; $\mathrm{FN}=$ false negative; $\mathrm{FP}=$ false positive; $n=$ number of samples in the test set; $k=$ Number of classes

\section{RESULTS AND DISCUSSION}

In this section, we discuss the overall model performance, the effect of layer count on model performance, and the effect of image splitting on model performance.

\subsection{Overall performance of the models}

Table 2 shows the performance of each classification model built against the original full-size dataset as well as the modified dataset with the image broken down into smaller sizes. On the original dasaset, the best performance was shown by densenet 169 with an accuracy of $79.17 \%$, precision of $80.98 \%$, recall of $79.17 \%$ and F-measure of $79.03 \%$. Meanwhile, the worst performance is shown by vgg 11 _bn with accuracy, precision, recall, and F-measure below 50\%, only slightly above the random baseline classifier $(33.33 \%)$. In the modified dataset, the greatest performance was achieved by vgg13_bn, with an accuracy of $87.61 \%, 88.62 \%$ precision, $87.81 \%$ recall, and $87.32 \%$ F-measure which were the highest values for each 
performance metric. In general, all models perform fairly well, with above $75 \%$ performance across all models and measurement metrics.

Table 2. The performance of each model on the original and modified dataset

\begin{tabular}{ccccccccc}
\hline \multirow{2}{*}{ Model } & \multicolumn{3}{c}{ Original Dataset } & \multicolumn{4}{c}{ Modified Dataset } \\
\cline { 2 - 8 } & Accuracy & Precision & Recall & F-Measure & Accuracy & Precision & Recall & F-Measure \\
\hline resnet18 & 0.6917 & 0.7030 & 0.6917 & 0.6868 & 0.7794 & 0.7932 & 0.7794 & 0.7745 \\
resnet34 & $\mathbf{0 . 7 5 0 0}$ & 0.7635 & $\mathbf{0 . 7 5 0 0}$ & $\mathbf{0 . 7 4 1 9}$ & 0.7981 & 0.8077 & 0.7981 & 0.7956 \\
resnet50 & 0.7083 & 0.7337 & 0.7083 & 0.7080 & 0.8303 & 0.8434 & 0.8303 & 0.8282 \\
resnet101 & 0.7167 & 0.7263 & 0.7167 & 0.7136 & 0.8347 & 0.8610 & 0.8347 & 0.8317 \\
resnet152 & 0.6917 & 0.7324 & 0.6917 & 0.6891 & 0.8408 & 0.8535 & 0.8408 & 0.8398 \\
densenet121 & 0.6833 & 0.6812 & 0.6833 & 0.6654 & $\mathbf{0 . 8 5 5 0}$ & $\mathbf{0 . 8 6 6 0}$ & $\mathbf{0 . 8 5 5 0}$ & $\mathbf{0 . 8 5 1 3}$ \\
densenet161 & $\mathbf{0 . 7 5 0 0}$ & $\mathbf{0 . 8 0 0 8}$ & $\mathbf{0 . 7 5 0 0}$ & $\mathbf{0 . 7 3 8 0}$ & 0.8278 & 0.8420 & 0.8278 & 0.8271 \\
densenet169 & $\mathbf{0 . 7 9 1 7}$ & $\mathbf{0 . 8 0 9 8}$ & $\mathbf{0 . 7 9 1 7}$ & $\mathbf{0 . 7 9 0 3}$ & 0.7972 & 0.8089 & 0.7972 & 0.7910 \\
densenet201 & 0.7417 & $\mathbf{0 . 7 7 0 0}$ & 0.7417 & 0.7368 & 0.7989 & 0.8162 & 0.7989 & 0.7928 \\
vgg11_bn & 0.4417 & 0.4223 & 0.4417 & 0.4085 & 0.8481 & 0.8634 & 0.8481 & 0.8448 \\
vgg13_bn & 0.5917 & 0.5984 & 0.5917 & 0.5782 & $\mathbf{0 . 8 7 6 1}$ & $\mathbf{0 . 8 8 6 2}$ & $\mathbf{0 . 8 7 6 1}$ & $\mathbf{0 . 8 7 3 2}$ \\
vgg16_bn & 0.5250 & 0.4884 & 0.5250 & 0.4782 & $\mathbf{0 . 8 5 5 0}$ & $\mathbf{0 . 8 6 5 8}$ & $\mathbf{0 . 8 5 5 0}$ & $\mathbf{0 . 8 5 2 6}$ \\
vgg19_bn & 0.6500 & 0.6580 & 0.6500 & 0.6340 & 0.8392 & 0.8523 & 0.8392 & 0.8365 \\
\hline
\end{tabular}

\subsection{The effect of increasing the number of layers in the model on performance}

From Table 2, we can also see that there is no clear relationship between the number of layers in the model and the resulting performance. In the original dataset, DenseNet performance tends to get better with increasing layers. However, this does not apply to densenet201 where the performance actually decreases when compared to densenet169. The opposite occurs in the modified dataset, where the performance of DenseNet models tends to decrease as the number of layers in the model increases. The DenseNet variant with the least number of layer (densenet121) actually shows the best performance compared to other model variants in the modified dataset. ResNet also shows similar thing. In the modified dataset, ResNet's performance in terms of accuracy tends to increase as the number of layers increases. However, the same thing is not shown in the original dataset where the best performance is shown by resnet34. Whereas in VGG, model performance tends to fluctuate as the number of layers increases. Thus, we cannot conclude with certainty the relationship between the number of layers in the model and the resulting performance.

\subsection{Comparison of model performance on the original and modified datasets}

Next, we will look at the effect of splitting the image to form a new dataset on the performance generated by the classification model. Figure 6 shows the difference in the performance of the model built with the modified dataset against the performance of the model built with the original dataset. From this figure, it can be seen that almost all models show an increase in performance when compared to the model in the original dataset.

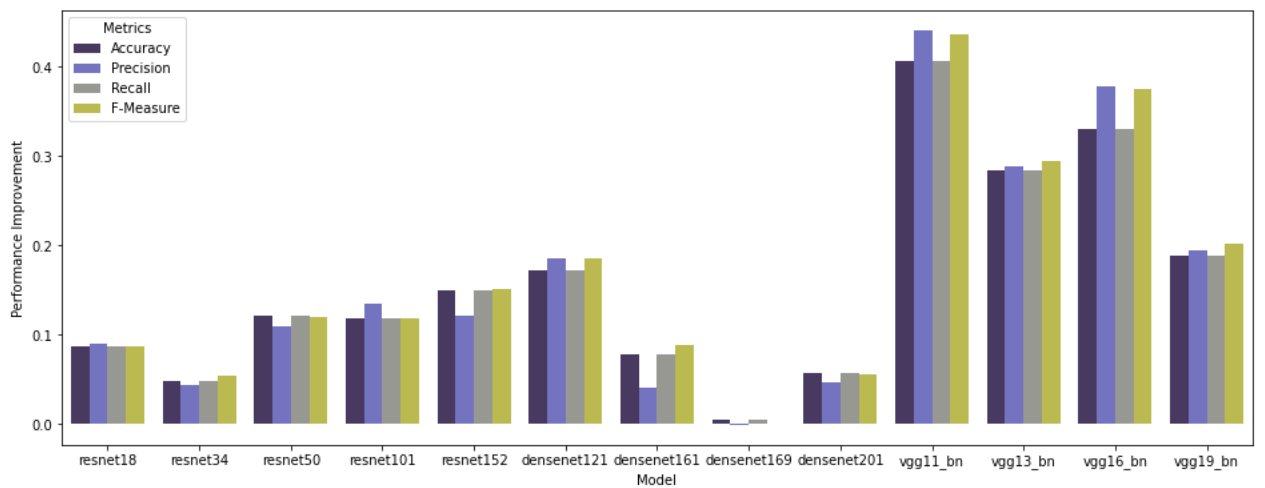

Figure 6. Difference in performance of models trained with the modified dataset against the models built using the original dataset

The highest increase is shown by VGG with one of its variants, namely vgg11_bn, showing a performance increase of around $100 \%$ in terms of accuracy, precision, recall, and F-measure. This is quite surprising considering that vgg11_bn was the model that performed the worst on the original dataset. In terms of performance improvement, ResNet is in second place where it can be seen from the graph that along 
with the increase in the number of layers, the performance improvement tends to increase. Whereas in Densenet the opposite happened where the increase in the number of layers was followed by a decrease in the performance increase. For densenet169, there is almost no difference in performance between the models built with the original dataset when compared to the models with the new dataset. This shows that in most architectures such as VGG and ResNet, the number and size of images have a very significant effect on the performance of the resulting model. Whereas in other architectures such as densenet169, the resulting model performance is not too sensitive to the number and size of training images. Further experiments are needed to investigate this.

\section{CONCLUSION}

From the results of this study, we have seen that $\mathrm{CNN}$ can be applied to the problem of identifying the method of batik making with a limited amount of data and has shown a fairly good performance. The best performance on native data is achieved by models with the densenet169 architecture. Whereas in the dataset with images broken down into smaller sizes, vgg13_bn shows the best performance. In most models, using a modified dataset will result in better performance when compared to using the original dataset. Although in certain variants such as densenet 169 , almost no change in performance was obtained from using the new dataset, which shows that densenet169 is not very sensitive to the number and size of images. In future research, we will try to implement $\mathrm{CNN}$ for similar problems with a larger amount of data and try other approaches such as anomaly detection to solve this problem. We will also try to apply CNN to the multiclass classification problem where one batik cloth can be made with a combination of different manufacturing methods, such as a combination of writing and stamp, writing and printing, and so on.

\section{ACKNOWLEDGEMENTS}

This research was supported by Lembaga Penelitian dan Pengabdian Masyarakat (LPPM) Universitas Internasional Semen Indonesia (UISI).

\section{REFERENCES}

[1] "Indonesian Batik- intangible heritage-Culture Sector-UNESCO," [Online]. Available: https://ich.unesco.org/en/RL/indonesian-batik-00170 (accessed Dec. 03, 2019).

[2] N. Krevitsky, "Batik - Art and Craft," New York, NY, USA: Reinhold Publishing Corporation, 1964.

[3] I. M. Elliot, "Batik Fabled Cloth of Java," Hong Kong: Periplus Editions (HK) Ltd., 2004.

[4] Y. Seo and K. shik Shin, "Hierarchical convolutional neural networks for fashion image classification," Expert Systems with Applications, vol. 116, pp. 328-339, 2019, doi: 10.1016/j.eswa.2018.09.022.

[5] L. Fang, Y. Jin, L. Huang, S. Guo, G. Zhao, and X. Chen, "Iterative fusion convolutional neural networks for classification of optical coherence tomography images," Journal of Visual Communication and Image Representation, vol. 59, pp. 327-333, 2019, doi: 10.1016/j.jvcir.2019.01.022.

[6] R. Ptucha, F. Petroski Such, S. Pillai, F. Brockler, V. Singh, and P. Hutkowski, "Intelligent character recognition using fully convolutional neural networks," Pattern Recognition, vol. 88, pp. 604-613, 2019, doi: 10.1016/j.patcog.2018.12.017.

[7] A. Krizhevsky, "One weird trick for parallelizing convolutional neural networks," 2014. [Online]. Available: http://arxiv.org/abs/1404.5997, (Accessed: Dec. 04, 2019)

[8] A. G. Howard, Menglong Zhu, Menglong Zhu, Bo Chen, Dmitry Kalenichenko, Weijun Wang, Tobias Weyand et al., "MobileNets: Efficient Convolutional Neural Networks for Mobile Vision Applications," arXiv:1704.04861v1, 2017, Accessed: Aug. 20, 2019. [Online]. Available: http://arxiv.org/abs/1704.04861.

[9] M. Sandler, A. Howard, M. Zhu, A. Zhmoginov, and L. C. Chen, "MobileNetV2: Inverted Residuals and Linear Bottlenecks," 2018 IEEE/CVF Conference on Computer Vision and Pattern Recognition, Salt Lake City, UT, USA, 2018, pp. 4510-4520, doi: 10.1109/CVPR.2018.00474.

[10] K. He, X. Zhang, S. Ren, and J. Sun, "Deep residual learning for image recognition," 2016 IEEE Conference on Computer Vision and Pattern Recognition (CVPR), Las Vegas, NV, USA, 2016, pp. 770-778, doi: 10.1109/CVPR.2016.90.

[11] G. Huang, Z. Liu, L. van der Maaten, and K. Q. Weinberger, "Densely Connected Convolutional Networks," 2017 IEEE Conference on Computer Vision and Pattern Recognition (CVPR), Honolulu, HI, USA, 2017, pp. 2261-2269, doi: 10.1109/CVPR.2017.243.

[12] F. N. Iandola, S. Han, M. W. Moskewicz, K. Ashraf, W. J. Dally, and K. Keutzer, "SqueezeNet: AlexNet-level accuracy with 50x fewer parameters and $<0.5 \mathrm{MB}$ model size," ICLR 2017, 2017.

[13] K. Simonyan and A. Zisserman, "Very deep convolutional networks for large-scale image recognition," ICLR 2015, 2015. 
[14] I. Nurhaida, R. Manurung, and A. M. Arymurthy, "Performance comparison analysis features extraction methods for Batik recognition," 2012 International Conference on Advanced Computer Science and Information Systems (ICACSIS), Depok, West Java, Indonesia, 2012, pp. 207-212.

[15] I. Nurhaida, A. Noviyanto, R. Manurung, and A. M. Arymurthy, "Automatic Indonesian's Batik Pattern Recognition Using SIFT Approach,” Procedia Computer Science, vol. 59, pp. 567-576, 2015, doi: 10.1016/j.procs.2015.07.547.

[16] I. Nurhaida, H. Wei, R. A. M. Zen, R. Manurung, and A. M. Arymurthy, "Texture fusion for batik motif retrieval system," International Journal of Electrical and Computer Engineering (IJECE), vol. 6, no. 6, pp. 3174-3187, 2016, doi: 10.11591/ijece.v6i6.12049.

[17] I. Setyawan, I. K. Timotius, and M. Kalvin, "Automatic batik motifs classification using various combinations of SIFT features moments and k-Nearest Neighbor," 2015 7th International Conference on Information Technology and Electrical Engineering (ICITEE), Chiang Mai, Thailand, 2015, pp. 269-274, doi: 10.1109/ICITEED.2015.7408954.

[18] A. E. Minarno, A. S. Maulani, A. Kurniawardhani, F. Bimantoro, and N. Suciati, "Comparison of methods for Batik classification using multi texton histogram," TELKOMNIKA (Telecommunication Computing Electronics and Control), vol. 16, no. 3, pp. 1358-1366, 2018, doi: 10.12928/TELKOMNIKA.v16i3.7376.

[19] A. A. Kasim, R. Wardoyo, and A. Harjoko, "The selection feature for batik motif classification with information gain value," International Conference on Soft Computing in Data Science-SCDS 2017, 2017, vol. 788, pp. $106-$ 115, doi: 10.1007/978-981-10-7242-0_9.

[20] A. A. Kasim, R. Wardoyo, and A. Harjoko, "Batik Classification with Artificial Neural Network Based on TextureShape Feature of Main Ornament," International Journal of Intelligent Systems and Applications, vol.9, no. 6, pp. 55-65, 2017, doi: 10.5815/ijisa.2017.06.06

[21] R. Azhar, D. Tuwohingide, D. Kamudi, Sarimuddin, and N. Suciati, "Batik Image Classification Using SIFT Feature Extraction, Bag of Features and Support Vector Machine,” Procedia Computer Science, vol. 72, pp. 24-30, 2015, doi: 10.1016/j.procs.2015.12.101.

[22] Farida, R. E. Caraka, T. W. Cenggoro, and B. Pardamean, "Batik Parang Rusak Detection Using Geometric Invariant Moment," 2018 Indonesian Association for Pattern Recognition International Conference (INAPR), Jakarta, Indonesia, 2018, pp. 71-74, pp. 71-74, doi: 10.1109/INAPR.2018.8627000.

[23] C. Jatmoko and D. Sinaga, "A Classification of Batik Lasem using Texture Feature Ecxtraction Based on KNearest Neighbor," Journal of Applied Intelligent System, vol. 3, no. 2, pp. 96-107, 2019, doi: 10.33633/jais.v3i2.2151.

[24] N. Yunari, E. M. Yuniarno, and M. H. Purnomo, "Indonesian batik image classification using statistical texture feature extraction Gray Level Co-occurrence Matrix (GLCM) and Learning Vector Quantization (LVQ)," Journal of Telecommunication, Electronic and Computer Engineering, vol. 10, no. 2-3, pp. 67-71, 2018.

[25] F. U. Karimah and A. Harjoko, "Classification of batik kain besurek image using speed up robust features (SURF) and gray level co-occurrence matrix (GLCM)," International Conference on Soft Computing in Data Science-SCDS 2017, 2017, vol. 788, pp. 81-91, doi: 10.1007/978-981-10-7242-0_7.

[26] W. Herulambang, M. N. Hamidah, and F. Setyatama, "Comparison of SVM and BPNN Methods in the Classification of Batik Patterns Based on Color Histograms and Invariant Moments," 2020 International Conference on Smart Technology and Applications (ICoSTA), Surabaya, Indonesia, 2020, pp. 1-4, doi: 10.1109/ICoSTA48221.2020.1570615583.

[27] A. E. Minarno, Y. Azhar, F. D. Setiawan Sumadi, and Y. Munarko, "A Robust Batik Image Classification using Multi Texton Co-Occurrence Descriptor and Support Vector Machine," 2020 3rd International Conference on Intelligent Autonomous Systems (ICoIAS), Singapore, 2020, pp. 51-55, doi: 10.1109/ICoIAS49312.2020.9081833.

[28] A. E. Minarno, F. D. S. Sumadi, H. Wibowo, and Y. Munarko, "Classification of batik patterns using K-nearest neighbor and support vector machine," Bulletin of Electrical Engineering and Informatics (BEEI), vol. 9, no. 3, pp. 1260-1267, 2020, doi: 10.11591/eei.v9i3.1971.

[29] I. M. A. Agastya and A. Setyanto, "Classification of Indonesian batik using deep learning techniques and data augmentation," 2018 3rd International Conference on Information Technology, Information System and Electrical Engineering (ICITISEE), Yogyakarta, Indonesia, 2018, pp. 27-31, doi: 10.1109/ICITISEE.2018.8720990.

[30] A. Y. Wicaksono, N. Suciati, C. Fatichah, K. Uchimura, and G. Koutaki, "Modified Convolutional Neural Network Architecture for Batik Motif Image Classification," IPTEK, Journal of Science, vol. 2, no. 2, pp. 26-30, 2017, doi: 10.12962/j23378530.v2i2.a2846.

[31] Y. Gultom, A. M. Arymurthy, and R. J. Masikome, "Batik Classification using Deep Convolutional Network Transfer Learning," Jurnal Ilmu Komputer dan Informasi, vol. 11, no. 2, pp. 59-66, 2018, doi: 10.21609/jiki.v11i2.507.

[32] H. Prasetyo and B. A. Putra Akardihas, "Batik image retrieval using convolutional neural network," TELKOMNIKA (Telecommunication Computing Electronics and Control), vol. 17, no. 6, pp. 3010-3018, 2019, doi: 10.12928/TELKOMNIKA.v17i6.12701.

[33] J. Tristanto, J. Hendryli, and D. Erny Herwindiati, "Classification of Batik Motifs Using Convolutional Neural Networks," International Conference on Information Technology, Engineering, Science \& its Applications, pp. 1-5, 2018, doi: $10.2139 / \mathrm{ssrn} .3258935$.

[34] M. A. Rasyidi and T. Bariyah, "Batik pattern recognition using convolutional neural network," Bulletin of Electrical Engineering and Informatics (BEEI), vol. 9, no. 4, pp. 1430-1437, 2020, doi: 10.11591/eei.v9i4.2385.

[35] L. N. Smith, "A disciplined approach to neural network hyper-parameters: Part 1-learning rate, batch size, momentum, and weight decay," arXiv:1803.09820, 2018. 